\title{
Anuros del gradiente altitudinal bosque altoandino-subpáramo en un sector de los Farallones del Citará
}

\section{Anuros of the gradient altitudinal forest Andean-sub-paramo in a sector of the cliffs of the quote}

\author{
Jimi Moya-Robledo*
}

\section{Resumen}

Se evalúo la fauna de anuros que ocurre en un gradiente altitudinal del bosque alto andino-subpáramo; el rango altitudinal estuvo estipulado entre 3.100 y 3.700 msnm. Durante el estudio se establecieron siete estaciones de trabajo, cada una separada de la anterior y siguiente por 100 metros de altitud. El método de muestreo fue la búsqueda libre sin restricciones; los ejemplares colectados fueron fotografiados y preparados según el método de Simmons (1987); se determinaron las diversidades alfa y beta con el fin de establecer la riqueza específica de la localidad, además se realizaron dendrogramas de similitud Jaccard para mirar la afinidad entre las especies y estaciones de trabajo. El estudio arrojo el registro de siete morfoespecies todas pertenecientes al género Pristimantis, además de la confirmación del recambio de especies a lo largo del gradiente altitudinal así como la ocupación de nichos tróficos a medida que este aumenta. Se evidenció de igual forma que en las estaciones tres y cuatro a 3.300 y 3.400 msnm (donde se registró la mayor riqueza específica de anuros), existe la particularidad que sobre este tramo del gradiente, se conjugan elementos de bosque alto andino y subpáramo que eventualmente podrían brindar diversas oportunidades de hábitat y anclaje para soportar una mayor cantidad de especies.

Palabras clave: Anuros, Farallones del Citará, Páramo, Subpáramo.

\begin{abstract}
I evaluate the fauna of anuros that happens in a gradient altitudinal of the high Andean forest-Subhigh plateau, the range altitudinal was stipulated between 3.100 and 3.700 msnm; during the study there were established seven stations of sampling, each one separated of previous and following by 100 meters of alti-
\end{abstract}

* Investigador Asociado II, Instituto de Investigaciones Ambientales del Pacífico (IIAP), Quibdó, Chocó. e-mail: jmoya@iiap.org.co 
tude. The method of sampling was the free search without restrictions, the collected copies were photographed and prepared according to the method of Simmons (1987), the diversities decided alpha and thread in order to establish the wealth specifies of the locality. In addition they were realized dendrogramas of similarity Jaccard to look at the affinity between the species and working stations. The study I throw the record of seven morfospice all the belonging ones to the kind Pristimantis. Besides the confirmation of the refill of species along the gradient altitudinal as well as the occupation of niches tróficos as it increases this one. I demonstrate of equal form that on the stations three and four to 3.300 and 3.400 msnm (where the major wealth was registered it specifies of anuros), exists the particularity that in this transecto of the gradient there conjugate elements of high Andean forest and sub high plateau that eventually there might offer diverse opportunities of habitat and anchorage to support major a major quantity of species.

Keywords: Anuran, Farallons of the Citará, Paramo, Subparamo.

\section{Introducción}

La distribución de los organismos a lo largo de un gradiente altitudinal tiende a permanecer como uno de los más interesante tópicos biogeográficos, porque las características físicas (temperatura, precipitación, presión atmosférica, entre otras) asociadas con la altitud, cambian drásticamente afectando a la diversidad y distribución de las especies, incluso en cortas distancias (Yu 1994). Wallace (1878) reconoció que la vida animal era un conjunto más abundante y variable en los trópicos que en otras partes del globo y que no era posible dar una cifra exacta del número de especies existentes en la Tierra, estableciéndose que los trópicos contienen más especies que en latitudes más altas (Rohde1992). Asimismo, se ha determinado que en muchos grupos de vertebrados la riqueza de especies y la abundancia relativa varían con la altitud (Diamond 1972, Terborgh 1977, Graham 1983, Heaney et al. 1989). La declinación de la riqueza de especies con el incremento de la altura está ampliamente aceptada como patrón general (Terborgh 1971, Terborgh y Weske 1975, Terborgh 1977, Koopman 1978, Duellman 1979, Graham 1983, Duellman 1988). Según lo anotado por (Duellman 1988, Duellman 1999, Duellman y Trueb 1986, Lynch y Duellman 1980, Navas 1999, 2002 y Vaira 2001) esta disminución es común en los anfibios y reptiles que habitan los gradientes altitudinales de los Andes y puede presentarse de manera uniforme (Heatwole 1982) o con un pico máximo de riqueza a alturas intermedias (500-1900 m) (Heyer 1967, Lynch y Duellman1997, Suárez-Mayorga 1999); sin embargo, tal diversidad es taxa específica y está relacionada con una fisiología termal muy lábil que presentan los anuros (Navas 2002).

Según lo anterior y tomando en cuenta los patrones de variación sistémica del grupo, se evaluó la diversidad de anuros en el bosque alto andino subpáramo (3.100-3.700 msnm), esperando aportar a las microvariaciones taxonómicas identificadas a lo largo de la franja de transición de estos dos ecosistemas.

Área de estudio. Obedece a zonas de bosque altoandino y subpáramo ubicado entre 3.100 y $3.700 \mathrm{msnm}$, en los sectores conocidos como: La Virgen, La Señal y La Avioneta; el área de trabajo se caracteriza por ser sumamente escarpada con pendientes que alcanzan los 80 grados de inclinación y presentar la vegetación típica de los bosque altoandinos y subpáramo donde predominan entidades florísticas como Melastomataceae, Ericaceae, Clusiaceae, Lauraceae y Cunoniaceae, destacándose además una gran abundancia de bromelias, chuscales y pajonales que sirven de anclaje a la anurofauna local. 


\section{Bioetnia Volumen 13, 2016}

\section{Métodos}

Por la complejidad del gradiente y lo escarpado del complejo Farallones del Citará, se estableció el mecanismo de muestreo de búsqueda libre sin restricciones, para lo cual se establecieron siete estaciones de trabajo ubicadas entre 3.100 y 3.700 msnm; los muestreo en las estaciones se realizaron a través de caminatas diurnas y nocturnas, con un trabajo intensivo de entre 20 a 40 minutos de duración, utilizando el método de búsqueda libre sin restricciones a través de exploraciones en todos los microhábitats disponibles.

Dentro de los tipos de hábitats y microhábitats presentes en la zona, se registraron todas las especies así como los datos ecológicos relevantes sobre rocas, troncos caídos, bordes de quebradas, colchones de hepáticas, macollas, bajo-entre y sobre necromasa de frailejones y puya, troncos de árboles y arbustos, sobre el suelo cubierto por hojarasca o desnudo, entre vegetación rasante, dentro de pozos y quebradas, entre otros; además de utilizar en conjunto la técnica de remoción con rastrillo(Mueses-Cisneros y Yañez-Muñoz 2009, Salinas y Veintimilla 2010), donde en cada estación de trabajo mediante el uso de una herramienta de labranza se removieron los troncos, necromasa de frailejones y macollas, y mantillo del suelo.

Sacrificio, preparación y fijación. La preparación de los individuos capturados como especímenes científicos, se realizó a través de la aplicación de xilocaína gel en el dorso de los organismos, para el sacrificio; luego se fijaron en una posición ya preestablecida en una bandeja con formol al 10\% y por último se etiquetan los individuos (Simmons 1987, Mc Diarmid 2001).

Toma de datos (ambientales y estructurales). Para cada individuo colectado se tomaron datos como: coordenadas geográficas por estación de muestreo, altitud, fotografías, actividad (D: diurno, N: nocturno), hora de captura, ambiente terrestre o acuático, hábito: terrestre, acuático, arborícola o fosorial, microhábitat donde se capturó: bajo y sobre rocas, troncos caídos, bordes de quebradas, colchones de hepáticas, macollas, bajo, entre y sobre necromasa de frailejones y puya, troncos de árboles y arbustos, sobre el suelo cubierto por hojarasca o desnudo, entre vegetación rasante, dentro de pozos y quebradas, entre otros.

Análisis de datos. Se evaluó la diversidad alfa como la riqueza o número total de especies. Se estimó la representatividad del muestreo mediante la construcción de una curva de acumulación de especies a partir de los estimadores de riqueza no paramétricos (Colwell y Coddington 1994, Moreno 2001, Magurran 2004) con el fin determinar si disminuyeron las especies al aumentar el esfuerzo de muestreo.

Para identificar la franja de transición entre bosque y páramo, se compararon con respecto a la diversidad beta, las estaciones de muestreo que deben estardistribuidas a lo largo de un gradiente de condiciones ecológicas. Para este fin, se construyó una matriz de presencia-ausencia o abundancia, con base en los datos de campo de las especies colectadas, para lo cual se usó una matriz dependiendo de la cantidad de datos que se obtuvieron en los muestreos; es decir, para un conjunto de datos relativamente pequeño se usó una medida de similitud que se basó en presencia-ausencia. Como fin se comprendieron las relaciones de similitud entre las estaciones de muestreo, para lo cual se realizó un análisis de agrupamiento usando como medida de similitud el índice de Jaccard.

Especies endémicas vulnerables o de valor comercial. Para la determinación de especies endémicas se tuvo en cuenta el rango de distribución de las especies; las vulnerables o amenazadas se tomaron a través de listados de la UICN (Unión Internacional para la Conservación de la Naturaleza) y la Resolución Nº 0192 de 2014 del MAVDS; el valor comercial se determinó basado de acuerdo con el uso y la relación de los habitantes locales con estos organismos. 


\section{Resultados y discusión}

Composición y estructura. Durante el tiempo de trabajo de campo en el gradiente altitudinal bosque alto andino-subpáramo, se registraron siete especies de anfibios todas pertenecientes a la familia Craugastoridae y el género Pristimantis que involucra las reconocidas ranitas de lluvia; estas obedecen al grupo más representativo de la anfibiofauna alto andina y paramuna de los Andes colombianos. Este género a su vez es el más abundante en el neotrópico (Lynch, 1998, Rincón-Franco y Castro 1998) y el género de vertebrados terrestres más abundante por su estrategia reproductiva en ausencia de cuerpos de agua y desarrollo directo. El 80\% de las especies de alta montaña pertenecen a este género que posee estrategias de huevos no acuáticos, lo que explica su alta diversidad. El total de las siete morfoespecies (Pristimantis) indican el mismo número de diversidad alfa (N:7), es decir, que obedecen al número total encontrado sobre las siete estaciones establecidas para del gradiente altitudinal (3.100 a $3.700 \mathrm{msnm}$ ).

La riqueza específica a lo largo del gradiente, se distribuyó de manera diferente entre estaciones: para la primera estación de trabajo (correspondiente a la estación siete ubicada sobre $3.700 \mathrm{msnm}$ ), se colectó un solo individuo identificado como
Pristimantis sp 3, en la estación 6 a $3.600 \mathrm{msnm}$ (N $\left.05^{\circ} 33^{\prime} 02^{\prime \prime} \mathrm{W} 075^{\circ} 58^{\prime} 55.7^{\prime \prime}\right)$; se colectaron dos especies Pristimantis sp 1 y Pristimantis $s p$ 2, en la estación 5 a $3.500 \mathrm{msnm}$ (N 05 33' 23.3" W $075^{\circ} 33^{\prime} 20.3^{\prime \prime}$ ) solo se registró Pristimantis sp 1 , que es además la más común en el gradiente altitudinal entre 3.300 y $3.600 \mathrm{msnm}$; mientras que para la estación 4 en el sitio conocido como La Señal (3.400 msnm) se registraron cuatro Pristimantis ( $s p 1, s p 2, s p 4$ y $s p$ 5). En la estación 3 ( $\left.3.300 \mathrm{msnm} \mathrm{N} 05^{\circ} 33^{\prime} 24.3^{\prime \prime} \mathrm{W} 075^{\circ} 58^{\prime} 47.6^{\prime \prime}\right)$ se colectaron tres especímenes correspondientes a los morfos Pristimantis sp 1 sp 4 y sp 5, mientras que en las estaciones 1 y 2 ( $3.100 \mathrm{~N} 05^{\circ} 33^{\prime} 38.5^{\prime \prime}$ $\mathrm{W} 075^{\circ} 58^{\prime} 20.9^{\prime \prime}$ y $3.200 \mathrm{msnm} \mathrm{N} 05^{\circ} 33^{\prime} 36.6^{\prime \prime}$ $\mathrm{W} 075^{\circ} 58^{\prime} 32.0^{\prime \prime}$ respectivamente), se colectaron los morfos correspondientes a Pristimantis sp 6 y sp 7 (Tabla 1 y Figura 1).

Las estaciones ubicadas entre 3.100 y 3.200 msnm (bosque alto andino) registraron dos especies con una conducta diferente a las encontradas en las demás estaciones; estas especies denominadas Pristimantis sp 6 y $s p$ 7, presentaron un comportamiento asociado con la supervivencia entre las axilas de bromelias que fue variando en medida que aumentó el gradiente para lo cual entre $3.300 \mathrm{msnm}$ en adelante se ocuparon las hojas de la vegetación típica y en medida que se ascendió hacia los 3.400, donde comienza la

Tabla 1. Composición de anuros en un gradiente altitudinal del cerro La Laguna, Farallones del Citará

\begin{tabular}{|c|c|c|c|c|c|c|c|}
\hline $\begin{array}{l}\text { Estaciones de trabajo/ } \\
\text { msnm } \\
\text { Especies registradas }\end{array}$ & 3.100 (E.1) & 3.200 (E.2) & 3.300 (E.3) & 3.400 (E.4) & 3.500 (E.5) & 3.600 (E.6) & 3.700 (E.7) \\
\hline Pristimantis sp 1 & & & $\mathrm{x}$ & $\mathrm{x}$ & $\mathrm{x}$ & $\mathrm{x}$ & \\
\hline Pristimantis sp 2 & & & & $\mathrm{x}$ & & $x$ & \\
\hline Pristimantis sp 3 & & & & & & & $x$ \\
\hline Pristimantis sp 4 & & & $x$ & $\mathrm{x}$ & & & \\
\hline Pristimantis sp 5 & & & $\mathrm{x}$ & $\mathrm{x}$ & & & \\
\hline Pristimantis sp 6 & $\mathrm{x}$ & $\mathrm{x}$ & & & & & \\
\hline Pristimantis sp 7 & $\mathrm{x}$ & $\mathrm{x}$ & & & & & \\
\hline
\end{tabular}




\section{Bioetnia Volumen 13, 2016}

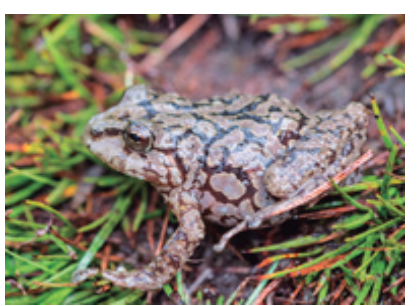

Pristimantis sp 1

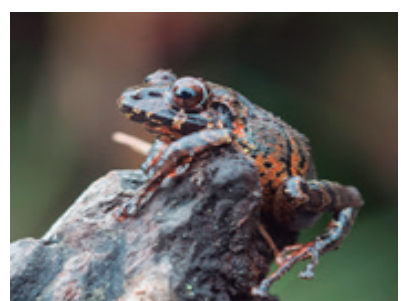

Pristimantis sp 3

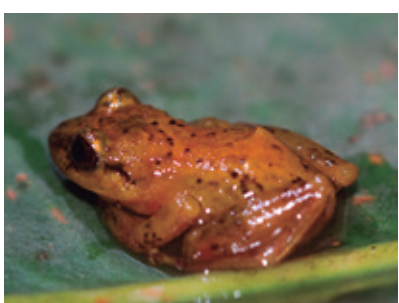

Pristimantis sp 6

Figura 1. Registro fotográficos de algunos individuos de Pristimantis colectados en un gradiente del cerro La Laguna.

aparición del frailejón, se empiezan a observan individuos ocupando estos nichos.

La diversidad de Pristimantis es quizás lo esperado porque este género es el más diverso en ecosistemas boscosos alto andinos de niebla y paramunos. Pefaur y Duellman (1980) y Lynch (1986) indican que en bosques de niebla subpáramo y páramo se encuentran 10 géneros de anuros con 48 especies de las cuales la mitad obedece a este género.

Recambio de especies. La diversidad del género en los ecosistemas evaluados, permitió corroborar lo manifestado por múltiples autores, en donde se observa un recambio de los diferentes taxones a lo largo del gradiente como lo especifica la Tabla 1 donde se evidencia claramente como en la estaciones cambian las especies a partir de los $3.300 \mathrm{msnm}$, allí predominan Pristimantis $s p 1$, $s p 4$ y $s p 5$; estas se comparten y predominan en este tramo del gradiente al siguiente (E3 y E4); a partir de los $3.500 \mathrm{msnm}$ persiste Pristimantis sp $1 \mathrm{y}$ se mantiene hasta $3.600 \mathrm{msnm}$, donde nuevamente reaparece Pristimantis sp 2; luego se distinguió la aparición de una especie única en 3.700 msnm denominada Pristimantis sp 3.

En este sentido, este fenómeno podría ser atribuible a múltiples factores, entre estos se destaca principalmente que la composición y la estructura de los ensamblajes varió entre las estaciones a diferente altitud, lo cual ocasionó que en las formaciones de bosque altoandino predominara Pristimantis sp 6 y $s p 7$, mientras que en áreas de subpáramo predominaron las cinco especies restantes.
De otro lado, podría inferirse aun que los pocos registros obtenidos en los muestreos de bosque altoandino, no permiten establecer con certeza el nivel de este recambio de especies; no obstante, es de esperar que no todas las especies de anfibios en bosques altoandinos estén representadas en el ecosistema páramo, pues las condiciones de este último son adversas para la mayoría de anfibios. Al respecto Navas (2002), menciona que la temperatura y la radiación solar, entre otros aspectos abióticos, son factores que restringen la colonización de tierras altas por un mayor número de especies de anfibios porque afectan el desarrollo embrionario. Lynch y Suárez-Mayorga (2002) concluyen que la radiación solar, pero no la temperatura o la humedad, es un filtro para el establecimiento de anfibios en áreas abiertas de páramo pero no en áreas cerradas de bosque a menos que sean especies con características reproductivas específicas (p.e., posean huevos pigmentados y/o depositados en microhábitats protegidos).

Curvas de acumulación de especies. El análisis de la estructura de las curvas de diversidad señala que la comunidad de anfibios del gradiente sigue el patrón típico encontrado en otras comunidades en diversos ecosistemas. Los estimadores (Chao 1, Chao 2, ACE, ICE) presentan valores muy diferentes a los registrados en las especies observadas, por lo tanto es evidente que el esfuerzo de muestreo no fue suficiente para evaluar la diversidad que se espera para la zona; los estimadores a partir de la estación 6 tienden a presentar valores cercanos a los de las especies observadas. De igual manera, 


\section{Anuros y bosque altoandino-subpáramo. J Moya-Robledo}

la curva muestra que no se alcanzó la asíntota durante los tiempos de muestreo, por tanto se hace necesario emplear un mayor esfuerzo de muestreo mayor al empleado (Figura 2).

Índices de diversidad. Los índices de diversidad conforme el registro obtenido para todas las estaciones, sostienen que la riqueza, diversidad y dominancia varió significativamente a lo largo del gradiente, indicando que las estaciones $3 \mathrm{y}$ 4 presentaron la mayor riqueza de especies; estas dos estaciones además registraron la mayor abundancia, hecho atribuible a la disponibilidad de microambientes y a la heterogeneidad de elementos bióticos porque en este punto de las estaciones se da la transición entre el bosque alto andino y subpáramo lo que evidentemente refleja una mayor oportunidad de microambientes que es posible inciden en la presencia de estos taxas. Por cuanto a la dominancia se notó que fue mayor en las estaciones 5 y 7 , donde también se observó la menor riqueza, es decir una sola especie para cada estación, lo cual sugiere un atributo de dominio de estas especies en estos sectores del gradiente altitudinal 3.500 y $3.700 \mathrm{msnm}$. Contrario a lo que muestran los demás datos obtenidos para las otras estaciones, que comparten dos o más especies en común para estaciones contiguas, lo cual a su vez refleja una repartición equitativa de la muestra entre los taxas que ocurren en los demás sectores del gradiente altitudinal (Tabla 2).

Datos ambientalesy estructurales. Conforme la información ambiental y estructural, se encontró que las ranas que habitan el gradiente (bosque altoandino-subpáramo) son de hábitos exclusivamente nocturnos, por cuanto la presencia y actividad de estos organismos se registró durante

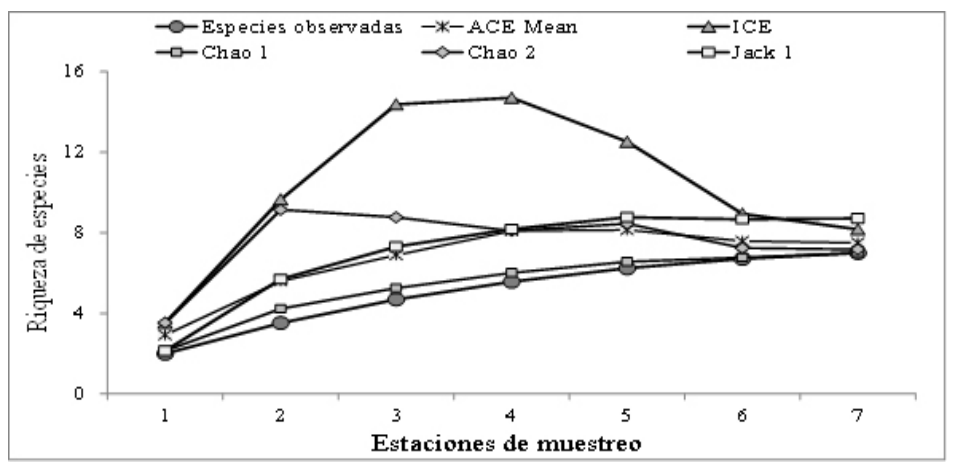

Figura 2. Curva de acumulación de especies.

Tabla 2. Índices de diversidad de especies

\begin{tabular}{lccccccc}
\hline Índices de diversidad & \multicolumn{7}{c}{ Estación } \\
\cline { 2 - 8 } & $\mathbf{1}$ & $\mathbf{2}$ & $\mathbf{3}$ & $\mathbf{4}$ & $\mathbf{5}$ & $\mathbf{6}$ & $\mathbf{7}$ \\
\hline Riqueza & 2 & 2 & 3 & 4 & 1 & 2 & 1 \\
Abundancia & 12 & 3 & 12 & 13 & 6 & 8 & 1 \\
Dominancia & 0.625 & 0.5556 & 0.5 & 0 & 1 & 0.7813 & 1 \\
Shannon-H & 0.5623 & 0.6365 & 0.8676 & 1.072 & 0 & 0.3768 & 0 \\
Menhinick & 0.5774 & 1.155 & 0.866 & 1.109 & 0.4082 & 0.7071 & 1 \\
Margalef & 0.4024 & 0.9102 & 0.8049 & 1.17 & 0 & 0.4809 & 0 \\
Fisher-alpha & 0.6853 & 2.622 & 1.284 & 1.974 & 0.3426 & 0.8559 & 0 \\
Berger-Parker & 0.75 & 0.6667 & 0.6667 & 1 & 1 & 0.875 & 1 \\
\hline
\end{tabular}


este horario de trabajo; en las horas del día no se obtuvo registro de individuos ni de ningún tipo de actividad vocal.

También se evidenció que todas las especies prefieren como sustrato de percha la vegetación disponible, en especial bromelias, chuscales, cicas y el frailejón, donde las ranas se perchan en un rango que va desde el suelo y oscila principalmente entre $40 \mathrm{~cm}$ y $1,60 \mathrm{~m}$ de altura, en estratos bajos como el suelo, necromasa y demás microambientes potenciales.

Diversidad beta. Dentro del gradiente se destacaron las estaciones ubicadas entre 3.400 y 3.700 msnm donde estas ranas aprovechan de manera frecuente las hojas de frailejón como sustrato de percha, que además son utilizadas por una variada gama de insectos (dípteros, hemíptera, himenóptera y lepidóptera) que también aprovechan este sustrato para percharse; mientras que en otras estaciones como las ubicadas en el subpáramo los organismos emplean como microhábitat las bromelias (Tabla 3 ) allí colectadas, es decir, que a medida que aumenta el gradiente,y la variabilidad en la vegetación es también diferente el uso de hábitat sobre todo a partir de los $3400 \mathrm{~m}$ de altura donde empieza a ser común el frailejón $\mathrm{y}$ en donde estos organismos lo prefieren como sustrato de percha.

Análisis clúster. Este análisis permitió observar claramente que las estaciones 1 y 2 fueron las más similiares entre sí (100\%) (Figura 3). Este resultado probablemente se relacione con el hecho de que en este tramo del gradiente correspondiente al bosque Alto andino (3.100 y 3.200 msnm respectivamente); se registraron las mismas especies Pristimantis sp 6 y sp 7 proporcionalmente en similar número de abundancia. En las estaciones 3 y 4 se observó una similitud cercana al 75\%. En estas estaciones se compartieron los morfos Pristimantis sp 1, sp 4 y sp 5, atribuibles a la disposición de microambientes disponibles y el inicio de la paramización del gradiente lo que podría explicar la oportunidad de nichos y recursos tróficos disponibles. En contraste, en la estación 7 se presentó la menor similitud y esto obedece a que allí se encontró una especie única de Pristimantis sp 3 diferente a las encontradas en las demás estaciones; este aspecto que podría sugerir una nueva tendencia frente al recambio de especies en el gradiente a partir de este punto porque las observadas en otras estaciones no fueron avistadas ni escuchadas allí; esto obviamente requiere de un esfuerzo de muestreo mayor al empleado así como lo sugieren los estimadores

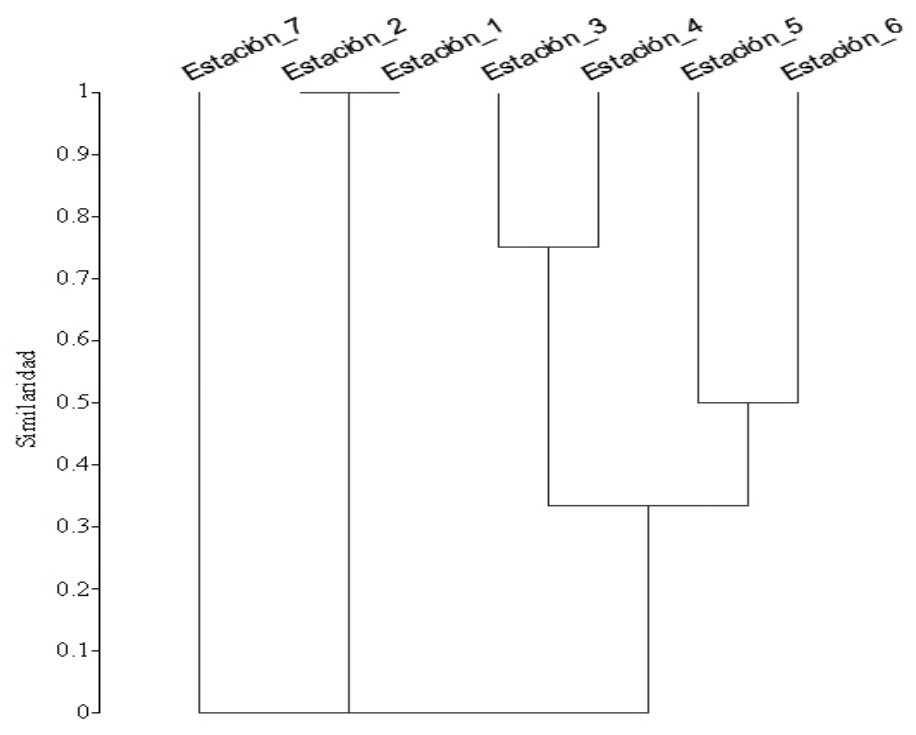

Figura 3. Análisis clúster entre estaciones de muestreo. 
Tabla 3. Microhabitats que ocupan las ranas de gradiente altitudinal bosque altoandino-subpáramo Farallones de Citará

\begin{tabular}{|c|c|c|c|c|c|c|}
\hline $\begin{array}{l}\text { Especies vs. } \\
\text { microhábitat }\end{array}$ & Macollas Necromasa & Frailejón Rocas Troncos caídos & Hojarasca & Suelo desnudo & Hojas & Bromelias \\
\hline Pristimantis sp 1 & & $\mathrm{x}$ & & $\mathrm{x}$ & $\mathrm{x}$ & \\
\hline Pristimantis sp 2 & & & & & $\mathrm{x}$ & \\
\hline Pristimantis sp 3 & & $\mathrm{x}$ & & & & \\
\hline Pristimantis sp 4 & & & & & $\mathrm{x}$ & \\
\hline Pristimantis sp 5 & & & & & $\mathrm{x}$ & \\
\hline Pristimantis sp 6 & & & & & & $\mathrm{x}$ \\
\hline Pristimantis sp 7 & & & & & & $\mathrm{x}$ \\
\hline
\end{tabular}

analizados a través de la curva de acumulación de especies.

Especies endémicas vulnerables o de valor comercial. Es muy probable que las especies encontradas a la fecha en este gradiente altitudinal sean propias de esta localidad por que presentan características únicas, además de ser un lugar bastante aislado de otros ecosistemas donde usualmente se han hecho inventarios más detallados. Afortunadamente ninguna de las especies Pristimantis se encuentra en listados de la UICN o la Resolución N³83; la accesibilidad a la zona es un limitante para desarrollar algún tipo de comercio con algunas de las ranas que habitan este ecosistema aislado de la cordillera Occidental.

\section{Consideraciones finales}

Los datos obtenidos están siendo prueba de análisis estadísticos y taxonómicos más detallados, con respecto a la obtención final de información comparable y edible entre lo estadístico y lo biológico; también se está trabajando para la designación e identificación taxonómica a nivel específico. Los resultados obtenidos sugieren que existe un recambio de especies a lo largo del gradiente altitudinal así como la ocupación de nichos tróficos a medida que aumenta el gradiente, siendo el punto más representativo en cuanto a riqueza específica las estaciones entre
3.300 y $3.400 \mathrm{msnm}$, donde empieza a denotarse la inherencia de la vegetación típica de páramo. A partir de 3.700 msnm se distinguió un morfo diferente que podría hacer pensar de la tendencia a encontrar otras especies a partir de este punto lo cual solo podría confirmarse con un esfuerzo de muestreo mayor al empleado como lo sugieren los estimadores utilizados en la curva de acumulación de especies.

\section{Literatura citada}

Colwell RK, Coddington JA. 1994. Estimating terrestrial biodiversity through extrapolation. Philos Trans R Soc Lonbd B Biol Sci. 345 (1311): 101-18. URL disponible en: https://www.ncbi.nlm.nih.gov/pubmed/7972351

Diamond JM. 1972. Avifauna of the Eastern highlands of New Guinea. Monograph of the Nattall Ornithological Club. Cambridge: Harvard University; 438 p. URL disponible en: https://sora.unm.edu/sites/default/files/ journals/auk/v091n01/p0192-p0210.pdf

Duellman WE (ed.). 1979. The herpetofauna of the Andes: patterns of distribution, origin, differentiation and present communities. En: The South American herpetofauna: its origin, evolution and dispersal. Mus Nat Hist University of Kansas, Monograph 7: 371-460. URL disponible en: https://archive.org/details/cbarchive 125864 theherpetofaunaoftheandespatte1979

Duellman WE, Trueb L. 1986. Biology of amphibians. New York, St. Louis, San Francisco: McGraw-Hill Co; $670 \mathrm{pp}$.

Duellman WE. 1988. Patterns of species diversity in anuran amphibians in the American tropics. Ann Missouri Bot Gard. 75 (1): 79-104. URL disponible en: https://www. 


\section{Bioetnia Volumen 13, 2016}

jstor.org/stable/2399467?seq=1\#page scan tab contents

Duellman WE. 1999. Distribution patterns of amphibians in South America. In: Patterns of distribution of amphibians. Baltimore and London: The Johns Hopkins University Press; pp. 255-327.

Fleming TH. 1986. Opportunism versus specialization: the evolution of feeding strategies in frugivorous bats. In: Estrada A, Fleming TH(eds.). Frugivores and seed dispersal. Dordrecht: Dr. W. Junk Publishers; pp. 105-18.

Graham GL. 1983. Changes in bat species diversity along an elevational gradient up the Peruvian Andes. J Mammal. 64 (4): 559-71. URL disponible en: https://academic.oup.com/jmammal/article-abstract/64/4/559/855749?redirectedFrom=fulltext

Heaney LR, Heideman PD, Rickart EA, Utzurrum RB, Klompen SH. 1989. Elevational zonation of mammals in the Central Philippines. J Trop Ecol. 5: 259-80. URL disponible en: https://www.cambridge.org/core/ journals/journal-of-tropical-ecology/article/elevational-zonation-of-mammals-in-the-central-philippines/521C0C7C8EFD2CDD65355BCAA90C2195

Heatwole H. 1982. A review of structuring in herpetofaunal assemblages. In: Scott Jr NJJ (ed.). Herpetological communities. A symposium of the Society for the Study ofAmphibians and Reptiles and the Herpetologist's League. 1977. Washintong, DC: Fish and Wildlife Research Report 13; 239 pp. URL disponible en: http://www4. ncsu.edu/unity/users/h/halfh/www/ecology.html

Heyer WR. 1967. A herpetofaunal study of an ecological transect through the cordillera de Tilaran, Costa Rica. Copeia. 2: 259-71. URL disponible en: https:/www. jstor.org/stable/1442113?seq=1\#page_scan_tab_con$\underline{\text { tents }}$

Koopman KF. 1978. Zoogeography of Peruvian bats with special emphasis on the role of the Andes. Am Mus Novit. 2651: 1-33. URL disponible en: http:// digitallibrary.amnh.org/bitstream/handle/2246/2965// v2/dspace/ingest/pdfSource/nov/N2651.pdf?sequence $=1$ \&isAllowed $=y$

Lynch JD, Duellman WE. 1980. The Eleutherodactylus of the Amazonian slopes of the Ecuadorian Andes (Anura: Leptodactylida). Miscellaneous publication. Lawrence: University of Kansas; pp. 1-86.

Lynch JD. 1986. Origins of the high Andean herpetological fauna. In: Viullecumier F, Monasterio M (eds). High altitud tropical biogeography. Oxford: University Press; pp. 478-99.

Lynch JD, Duellman WE. 1997. Frogs of the genus Eleutherodactylus (Leptodactylidae) in western Ecuador: systematics, ecology, and biogeography. Nat Hist Mus Univ Kansas Spec Publ. 23: 1-236.
Lynch JD. 1998. A new frog (genus Eleutherodactylus) from the cloud forests of southern Boyacá. Rev Acad Colomb Cienc. 22 (84): 429-32.

Lynch JD, Suárez-Mayorga AM. 2002. Reflexiones sobre el análisis biogeográfico de los anfibios paramunos. Memorias Congreso Mundial de Páramos. Memorias Tomo I; pp. 241-3. URL disponible en: http://bibdigital.rjb. csic.es/PDF/Fernandez_Paramos_Colombia_2002.pdf

Magurran AE. 2004. Measuring biological diversity. Blackwell Publishing; 256 pp.

McDiarmid RW. 2001. Preparación de anfibios como especímenes científicos. Pp 279-287. In: Heyer W, Donelly M, McDiarmid R, Hayek L, Foster M (eds.). Medición y monitoreo de la diversidad biológica, métodos estandarizados para anfibios. Washington y London: Smithsonian Institution Press; 349 pp.

Ministerio de Ambiente y Desarrollo Sostenible(MAVDS). 2014. Listado de las especies silvestres amenazadas de la diversidad biológica colombiana. Resolución $\mathrm{N}^{\circ}$ 0192 de febrero 10 de 2014. Bogotá: MAVDS. URL disponible en: http:/www.minambiente.gov.co/images/ normativa/resoluciones/2014/res 0192_2014.pdf

Moreno CE. 2001. Métodos para medir la biodiversidad. Rev Biol Trop. 49 (3-4): 1-84. URL disponible en: http://www.scielo.sa.cr/scielo.php?script=sci arttext\&pid=S0034-77442001000300090

Mueses-Cisneros JJ, Yañez-Muñoz MH. 2009. Anfibios. En: Vriesendorp C, Alverson WS, del Campo A, et al. (eds.). Ecuador: Cabeceras Cofanes-Chingual. Rapid Biological and Social Inventories Report 21. The Field Museum, Chicago. URL disponible en: http://fm2. fieldmuseum.org/rbi/pdfs/RI21 Spanish.pdf

Navas CA. 1999. Biodiversidad de anfibios y reptiles en el páramo: Una visión eco fisiológica. Rev Acad Colomb Cien Ex Fis Nat. 23: 465-74.

Navas CA. 2002. Herpetological diversity along Andean elevational gradients: links with physiological ecology and evolutionary physiology. Comparative Biochemistry and Physiology Part A: Molecular \& Integrative Physiology. 133 (3): 469-85. URL disponible en: https://www.sciencedirect.com/science/article/pii/ $\underline{\text { S1095643302002076 }}$

Pérfaur J, Duellman WE. 1980. Community structure in high Andean herpetofaunas. Trans Kansas Acad Sci. 83 (2): 45-65. URL disponible en: https://www.jstor. org/stable/3627715?seq=1\#page scan tab contents

Rohde K. 1992. Latitudinal gradients in species diversity: the search for the primary cause. Oikos. 65 (3): 514-27. URL disponible en: https://www.jstor.org/ stable/3545569?seq=1\#page scan tab contents

Simmons JE. 1987. Herpetological collection and collection management. Society for Study of Amphibians 


\section{Anuros y bosque altoandino-subpáramo. J Moya-Robledo}

and Reptiles.

Salinas KA, Veintimilla DA. 2010. Patrones de diversidad deAnuros en el ecosistema páramo del Parque Nacional Podocarpus. Tesis de grado Ingeniero en Manejo y Conservación del Medio Ambiente. Universidad Nacional de Loja. Ecuador. 99 pp. URL disponible en: http:// dspace.unl.edu.ec/jspui/bitstream/123456789/5180/1/ Salinas $\% 20$ Salinas $\% 20$ Karen $\% 20 \% 26 \% 20$ Veintimi1la $\% 20 \mathrm{Y} \% \mathrm{C} 3 \%$ A1nez $\% 20$ David.pdf

Suárez-MayorgaA. 1999. Lista preliminar de la fauna anfibia presente en el transecto La Montañita-Alto de Gabinete, Caquetá, Colombia. Rev Acad Colomb Cien Ex Fis Nat. 23: 395-405. URL disponible en: https://www. batrachia.com/orden-anura/hylidae/scinax-garbei/

Terborgh J. 1971. Distribution on environmental gradients: theory and a preliminary interpretation of distributional patterns in the avifaunaof the Cordillera Vilcabamba, Peru.Ecology. 52 (1): 23-40. URL disponible en: http:// onlinelibrary.wiley.com/doi/10.2307/1934735/full
Terborgh J, Weske JS. 1975. The role of competition in the distribution of Andean birds. Ecology. 56 (3): 562-76. URL disponible en: https://www.jstor.org/ stable/1935491?seq=1\#page scan tab contents

Terborgh J. 1977. Bird species diversity on an Andean elevational gradient. Ecology. 58 (5): 1007-19. URL disponible en: https://www.jstor.org/stable/1936921?seq=1\#page scan tab contents

Terborgh J. 1985. The role of the ecotones in the distribution of Andean Birds'. Ecology. 66 (4): 123746. URL disponible en: https://www.jstor.org/stable/1939177?seq=1\#page_scan tab_contents

Wallace AR. 1878. Tropical nature, and other essays. London: MacMillan and CO; 390 pp. URL disponible en: https://archive.org/details/tropicalnaturea00wallgoog

$\mathrm{Yu}$ H-T. 1994. Distribution and abundance of small mammals along a subtropical elevational gradient in central Taiwan. J Zool. 234 (4): 577-600. URL disponible en: http://onlinelibrary.wiley.com/ doi/10.1111/j.1469-7998.1994.tb04866.x/abstract 